\title{
Searches for short-baseline neutrino oscillations with the T2K off-axis near detector
}

\author{
Stefania Bordoni ${ }^{* \dagger}$ \\ Institut de Fìsica d'Altes Energies (IFAE), Barcelona (ES) \\ E-mail: sbordonilifae.es
}

The completeness of the three-neutrino mixing paradigm was challenged in the last 15 years. Indications of short baseline oscillations have been reported by several experiments. These may be explained by introducing one or more neutrinos that do not interact via the electroweak force: sterile neutrinos. The T2K off-axis near detector (ND280), thanks to its location at 280m from the proton target, can contribute to searches for short baseline neutrino oscillations. The T2K collaboration has recently published a first search for $v_{e}$ disappearance at ND280: at 95\% CL these results exclude the region $\sin ^{2} 2 \theta>0.2$ and $\Delta m^{2}>8 \mathrm{eV}^{2}$.

A new analysis looking for muon neutrino disappearance at ND280 is currently under development. The sensitivity study performed so far indicates that this analysis will have interesting results in 2015 .

XVI International Workshop on Neutrino Telescopes,

2-6 March 2015

Palazzo Franchetti, - Istituto Veneto, Venice, Italy

\footnotetext{
* Speaker.

${ }^{\dagger}$ On behalf of the T2K Collaboration
} 


\section{Introduction}

The completeness of the three-neutrino mixing paradigm has been challenged in the last 15 years. Indications of short baseline oscillations have been reported by a number of experiments in the $v_{e}$ channel. However, no hints of short baseline oscillations have been observed so far for muon neutrinos and tensions are observed between the existing experimental results[1].

The observed anomalies may be explained by introducing one or more sterile neutrinos: neutrinos that do not interact via the weak force. The existence of these new particles can be investigated by studying their mixing with the three standard neutrino flavours. The simplest theoretical model $(3+1$ model) considers the addition of only one sterile neutrino with a mass-squared difference $\Delta m_{41}^{2}$ of the order of $1 \mathrm{eV}^{2}$, thus much larger than the other mass differences. This makes the mass differences with respect to the standard neutrinos degenerate, so we can consider the survival probability in the two neutrino-state approximation to be valid:

$$
P\left(v_{\alpha} \rightarrow v_{\alpha}\right)=1-\sin ^{2}\left(2 \theta_{\alpha \alpha}\right) \cdot \sin ^{2}\left(\frac{1.267 \Delta m_{41}^{2}\left[e V^{2}\right] \mathrm{L}_{v}[\mathrm{~km}]}{E_{v}[G e V]}\right)
$$

where $\mathrm{L}_{v}$ and $\mathrm{E}_{v}$ are the neutrino oscillation distance and energy respectively. As is shown by Equation 1.1, for a given neutrino energy, a large value of $\Delta m_{41}^{2}$ would imply oscillation at short distance. The T2K off-axis near detector (ND280), thanks to its location at $280 \mathrm{~m}$ from the proton target offers good conditions to search for short baseline (SBL) neutrino oscillations.

\section{Analysis strategy}

To search for SBL oscillations a binned likelihood fit to the neutrino energy, reconstructed assuming Charged Current (CC) Quasi Elastic (QE) interactions, is performed. Such interactions are, in fact, dominant at the T2K energies. The dependency on the oscillation parameters $\left(\sin ^{2} 2 \theta\right.$ and $\Delta m_{41}^{2}$ ) is introduced by re-weighting the number of signal events by the survival probability (Eq. 1.1). The different sources of systematics are considered in the fit by including nuisance parameters with gaussian constraints, similar to what is done for other T2K analyses [2, 3].

The T2K collaboration has recently published the measurement of $v_{e}$ disappearance at ND280 [4]. A new analysis searching for muon neutrino disappearance is on-going. The poster presented here summarizes these two analyses.

\section{Short Baseline $v_{e}$ disappearance}

The electron neutrino component in the T2K neutrino beam is only about $1 \%$ of the total flux. However, a clean sample of $v_{e}$, with a purity of about $67 \%$ can be selected thanks to the very good performance of the TPCs and ECAL particle identification [5]. The main background for this analysis comes from Deep Inelastic scattering events and Neutral Current interactions where a $\pi^{0}$ is produced. Any photon coming from the decay of these $\pi^{0}$ can, in fact, be mis-identified as an electron produced by $v_{e}$-CC interactions. A control sample of photon conversion events with kinematics similar to the signal events is used to constrain this background. The search for $v_{e}$ disappearance has been performed using data collected between January 2010 and May 2013, 
corresponding to a total exposure of $6 \times 10^{20}$ protons on target (POT). The best fit oscillation parameters are $\sin ^{2} 2 \theta_{e e}=1$ and $\Delta m_{e f f}^{2}=2.05 \mathrm{eV}^{2} / \mathrm{c}^{4}$ is shown in Figure 1. Confidence intervals in the oscillation parameters phase space are computed using the Feldman-Cousins method. At $95 \% \mathrm{CL}$ we obtain an exclusion region for $\sin ^{2} 2 \theta_{e e}>0.3$ and $\Delta m_{e f f}^{2}>7 e V^{2} / c^{4}$. These results exclude a part of the gallium anomaly [6] and a small part of the reactor anomaly [7] allowed regions.

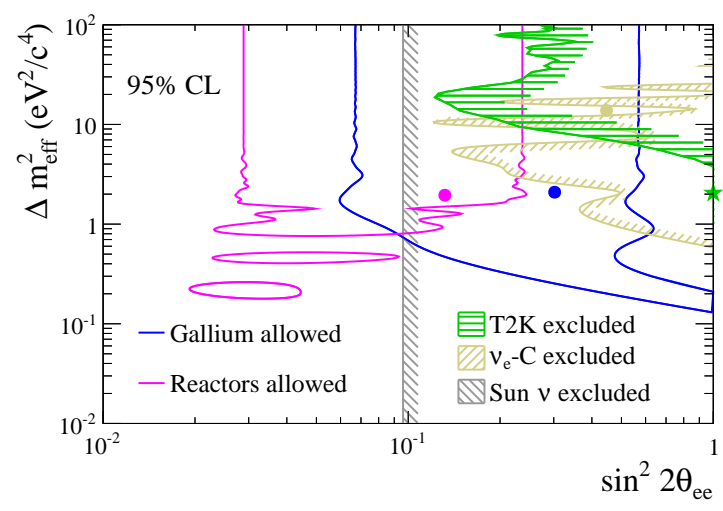

Figure 1: The T2K exclusion region at 95\% CL for the $v_{e}$ disappearance [4] compared with other experimental results. The T2K best fit is marked by a green star; the best fit of other experimental results corresponds to circles of the same coloring as the limits.

\section{Short Baseline $v_{\mu}$ disappearance}

The analysis is based on the muon neutrino CC interactions events selected using tracker informations (TPCs and FGDs). The selected events are then classified into three topologies by considering the number of final state pions: zero (CC0 $\pi$-like), one positive pion (CC1 $\pi$-like), and any other combination of number and charge (CCOther-like). Such classification allows a good control of the non-Quasi Elastic interactions, events which are characterised by higher energy than the $\mathrm{QE}$ events. Consequently, a better sensitivity in the high $\Delta m^{2}$ region is achieved.

The analysis presented in this poster describes a Monte Carlo based sensitivity study. The Monte Carlo simulation used for the study correspond to $3 \times 10^{20}$ POT and is normalised to the T2K statistics collected between January 2010 and May $2013\left(6 \times 10^{20}\right.$ POT). Figure 2 shows the sensitivity contours at $90 \% \mathrm{CL}$ which are estimated using a constant Delta Likelihood method. All dominant uncertainties are included and the obtained contours, compared to the existing limits [8] look promising, especially in the region at high $\Delta m^{2}$. Finalization of the current Monte Carlo studies are on-going and further developments aiming to the improve the current systematics are planned. The fit to data and the publication of those results are expected in the next few months.

\section{Conclusions}

In conclusion, the $\mathrm{T} 2 \mathrm{~K}$ collaboration is providing new and interesting results on the searches for short baseline neutrino oscillations. Results on the electron neutrino disappearance have been 


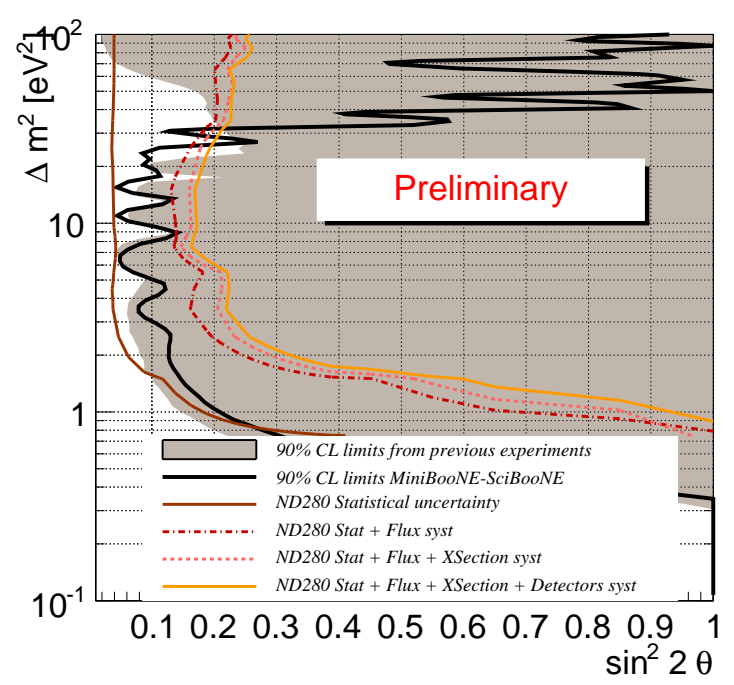

Figure 2: $\quad \mathrm{T} 2 \mathrm{~K}$ expected sensitivity to short baseline $v_{\mu}$ disappearance at $90 \% \mathrm{CL}$ with flux and crosssection and detector systematics included, compared to other experiments [8].

recently published and new results concerning the muon neutrino disappearance are foreseen in the next few months. The collection of further data will reduce the statistical uncertainty, which is an important limitation especially for the electron neutrino disappearance analysis. Finally, the analysis on muon neutrino disappearance is a necessary step to search for SBL electron neutrino appearance which is also predicted by the $3+1$ model. A joint analysis, aiming to fit electron and muon neutrino signals simultaneously to search for electron neutrino appearance, is foreseen for the near future.

\section{References}

[1] J Kopp et al., Sterile neutrino Oscillation: The Global Picture JHEP 1305:050 (2013)

[2] Abe et al., Observation of Electron Neutrino Appearance in a Muon Neutrino Beam, Phys Rev. Lett 112061802 (2014).

[3] Abe et al., Precise measurement of the neutrino mixing parameter $\theta_{23}$ from muon neutrino disappearance in an off-axis beam, Phys. Rev. Lett. 112181801 (2014).

[4] Abe et al., Search for short baseline $v_{e}$ disappearance with the T2K near detector, Phys. Rev. D. 91 $051102(2015)$

[5] Abe et al., Measurements of the intrinsic electron neutrino component in the T2K neutrino beam with the ND280 near detector, Phys RevD 89092003 (2014).

[6] F. Kaether et al., Reanalysis of the Gallex solar neutrino flux and source experiments, Physics Letters B 685 Issue 1 (2010).

[7] Mueller, Th. A. et al., Improved predictions of reactor antineutrino spectra, Phys. Rev. C 83054615 (2011).

[8] Mahn et al., Dual baseline search for muon neutrino disappearance at $0.5 \mathrm{eV}^{2}<\Delta m^{2}<40 \mathrm{eV}^{2}$, Phys. Rev. D 85032007 (2012). 